九州大学学術情報リポジトリ

Kyushu University Institutional Repository

\title{
A COMPUTATIONAL VERIFICATION METHOD OF SOLUTION WITH UNIQUENESS FOR OBSTACLE PROBLEMS
}

Ryoo, Cehon Seoung

Graduate School of Mathematics, Kyushu University

https://doi.org/10.5109/13475

出版情報: Bulletin of informatics and cybernetics. 30 (1)，pp.133-144，1998-03. Research Association of Statistical Sciences

バージョン :

権利関係 : 


\title{
A COMPUTATIONAL VERIFICATION METHOD OF SOLUTION WITH UNIQUENESS FOR OBSTACLE PROBLEMS
}

\author{
By \\ Cheon Seoung RYoo*
}

\begin{abstract}
A numerical method for automatic proof of the existence of solutions for variational inequalities is proposed. It is based on the infinite dimensional fixed point theorem and computable error estimates for finite element approximations of the original problems. Particularly, in this paper, we consider the method to prove the uniqueness of solution for obstacle problem. Further, some numerical examples are presented.
\end{abstract}

\section{Introduction}

In the author's previous work (Ryoo and Nakao (1998)), we proposed a numerical method for automatic proof of the existence of solutions for variational inequalities by computer. The basic approach of this method consists of the fixed point formulation of variational inequalities and the construction of the function set, in computer, satisfying the validation condition of infinite dimensional fixed point theorem, i.e., Schauder's fixed point theorem. In order to realize the verification, we used the finite element approximations and computable a priori error estimates as well as the method of interval arithmetic. In this paper, we consider the method to verify the local uniqueness of solution for obstacle problem. This method can be applied, at least theoretically, to general variational inequalities.

In the following section, we describe the obstacle problem considered in this paper and give the fixed point formulation to prove the existence of the solutions. In Section 3 , we introduce a computational verification condition. In Section 4, we present a computer algorithm, based upon the idea in the previous section, to construct the set satisfying the verification conditions by Schauder's fixed point theorem. Also we consider about the method to prove the uniqueness of solution for obstacle problem. Some numerical examples are illustrated in the last section.

\section{Problem and the fixed point formulation}

Let $\Omega$ be a bounded convex domain in $R^{n}, 1 \leq n \leq 2$, with piecewise smooth boundary $\partial \Omega$. We set $V \equiv H_{0}^{1}(\Omega)=\left\{v \in H^{1}(\Omega):\left.v\right|_{\partial \Omega}=0\right\}$ and

\footnotetext{
* Graduate School of Mathematics, Kyushu University 33, Fukuoka 812-81,Japan

This research was supported by Japan Society for the Promotion of Science, 1997.
} 


$$
a(w, v)=\int_{\Omega} \nabla w \cdot \nabla v d x
$$

where

$$
\nabla w \cdot \nabla v=\frac{\partial w}{\partial x_{1}} \frac{\partial v}{\partial x_{1}}+\frac{\partial w}{\partial x_{2}} \frac{\partial v}{\partial x_{2}} .
$$

We define $K=\{v \in V: v \geq 0$ a.e. on $\Omega\}$.

First, we note that, for any $g \in L^{2}(\Omega)$, the problem:

$$
a(w, \psi-w) \geq(g, \psi-w), \quad \forall \psi \in K, \quad w \in K,
$$

has a unique solution $w \in V \cap H^{2}(\Omega)$, and the estimate

$$
|w|_{H^{2}(\Omega)} \leq\|g\|_{L^{2}(\Omega)}
$$

holds (see Glowinski (1984) in detail), where $|w|_{H^{2}}$ implies the semi-norm of $w$ on $H^{2}(\Omega)$ defined by

$$
|w|_{H^{2}(\Omega)}^{2} \equiv \sum_{i, j=1}^{2}\left\|\frac{\partial^{2} w}{\partial x_{i} \partial x_{j}}\right\|_{L^{2}(\Omega)}^{2} .
$$

Now consider the following variational inequality;

$$
\left\{\begin{array}{l}
\text { Find } w \in K \quad \text { such that } \\
a(w, v-w) \geq(f(w), v-w), \forall v \in K
\end{array}\right.
$$

Here, $f$ is assumed to satisfy the following hypotheses :

A1. $f$ is the continuous map from $V$ to $L^{2}(\Omega)$.

A2. For each bounded subset $W \in V, f(W)$ is also bounded set in $L^{2}(\Omega)$.

We adopt $(\nabla \phi, \nabla \psi)$ as the inner product on $V$, where $(\cdot, \cdot)$ denotes the $L^{2}$ - inner product on $\Omega$. Hence, the associated norm is defined by $\|\phi\|_{V}=\|\nabla \phi\|_{L^{2}(\Omega)}$.

We now take an appropriate finite dimensional subspace $V_{h}$ of $V$ for $0<h<1$. Usually, $V_{h}$ is taken to be a finite element subspace with mesh size $h$. We then define $K_{h}$, an approximation of $K$, by

$$
K_{h}=V_{h} \cap K=\left\{v_{h} \mid v_{h} \in V_{h}, \quad v_{h} \geq 0 \text { on } \bar{\Omega}\right\} .
$$

We now define $v=P_{K}(w)$, the projection of $w \in V$ into $K$, as the solution of the following problem:

$$
v \in K: \quad a(v, \zeta-v) \geq a(w, \zeta-v), \quad \forall \zeta \in K
$$

Also define $v_{h}=P_{K_{h}}(w)$, the projection of $w$ into $K_{h}$, as follows:

$$
v_{h} \in K_{h}: \quad a\left(v_{h}, \zeta-v_{h}\right) \geq a\left(w, \zeta-v_{h}\right), \quad \forall \zeta \in K_{h} .
$$

Now, as one of the approximation properties of $K_{h}$, assume that 
A3. For each $w \in V \cap H^{2}(\Omega)$, there exists a positive constant $C$, independent of $h$, such that

$$
\left\|w-P_{K_{h}} w\right\|_{V} \leq C h|w|_{H^{2}(\Omega)} .
$$

Here, $C$ has to be numerically determined.

To verify the existence of a solution of (2.3) in a computer, we use the fixed point formulation. For each $w \in V$, by the Riesz representation theorem for the Hilbert space, there exists a unique $F(w) \in V$ such that

$$
(\nabla F(w), \nabla v)=(f(w), v), \quad \forall v \in V .
$$

That is,

$$
\left\{\begin{array}{c}
\exists F(w) \in V \text { such that } \\
-\Delta F(w)=f(w) \text { in } \Omega, \\
F(w)=0 \text { on } \partial \Omega .
\end{array}\right.
$$

Then the map $F: V \longrightarrow V$ is a compact operator.

By (2.7), problem (2.3) is equivalent to finding $w \in V$ such that

$$
a(w, v-w) \geq a(F(w), v-w), \quad \forall v \in K .
$$

By using (2.4) and (2.8), we now have the following fixed point problem for the compact operator $P_{K} F$.

Find $\exists w \in V$ such that $w=P_{K} F(w)$.

\section{Verification condition}

In order to deal with the functions and equations in the space $V$ of infinite dimension in a computer, we introduce two concepts, rounding and rounding error.

Now we define the dual cone of $K_{h}$ by

$$
K_{h}^{*}=\left\{w \in V: a(w, v) \leq 0, \quad \forall v \in K_{h}\right\}
$$

Note that $K_{h}^{*}$ is also closed convex cone in $V$ with vertex at 0 which is the only point common to $K_{h}$ and $K_{h}^{*}$. From (2.4) it follows that $K_{h}^{*}$ is the set of points whose projections into $K_{h}$ is 0 . We need some additional lemma which is from Rodrigues (1987).

Lemma 3.1. Any $w \in V$ can be uniquely decomposed into the sum of two orthogonal elements. That is,

$$
w=P_{K_{h}} w \bigoplus\left(I-P_{K_{h}}\right) w=P_{K_{h}} w \bigoplus P_{K_{h}^{*}} w
$$

Here, $\bigoplus$ denotes the sum of two orthogonal elements in the sense of $V$.

For any $w \in V$, we now define the rounding $R\left(P_{K} F(w)\right) \in K_{h}$ as the solution of the following problem:

$$
a\left(R\left(P_{K} F(w)\right), v_{h}-R\left(P_{K} F(w)\right)\right) \geq\left(f(w), v_{h}-R\left(P_{K} F(w)\right)\right), \quad \forall v_{h} \in K_{h}
$$


Next, for any subset $W \subset V$, we define the rounding $R\left(P_{K} F W\right) \subset K_{h}$ by the projection of $V$ onto $K_{h}$, that is,

$$
R\left(P_{K} F W\right)=\left\{w_{h} \in K_{h}: w_{h}=R\left(P_{K} F(w)\right), w \in W\right\} .
$$

Moreover, for $W \subset V$ we define $R E\left(P_{K} F W\right)$, the rounding error of $P_{K} F W$, as a subset of $K_{h}^{*}$, i.e.,

$$
R E\left(P_{K} F W\right)=\left\{v \in K_{h}^{*}:\|v\|_{V} \leq C h\|f(W)\|_{L^{2}}\right\},
$$

where

$$
\|f(W)\|_{L^{2}} \equiv \sup _{w \in W}\|f(w)\|_{L^{2}}
$$

Here, $C$ is the same positive constant as in (2.6). By using the approximation property A3 of $K_{h}$, we have

$$
P_{K} F(w)-R\left(P_{K} F(w)\right) \in R E\left(P_{K} F(w)\right), \quad \forall w \in W .
$$

Therefore, we have the following verification condition by Schauder's fixed point theorem.

LEMмA 3.2. If there exists a nonempty, bounded, convex, and closed subset $W \subset K$ such that

$$
R\left(P_{K} F W\right) \oplus R E\left(P_{K} F W\right) \subset W
$$

then there exists a solution of $w=P_{K} F(w)$ in $W$.

\section{Verification procedures with uniqueness}

In order to find a set $W$ satisfying the (3.2), we use iterative procedures, that is, the sequential iteration (see Ryoo and Nakao (1998)) or Newton-like method (see Nakao and Ryoo (1998)). In this paper, we describe only the former case for simplicity. In this section, we propose a computer algorithm to construct the set $W$ which satisfies the verification condition (3.2).

Now, we consider the following approximate problem corresponding to (2.1) as

$$
a\left(w_{h}, v_{h}-w_{h}\right) \geq\left(g, v_{h}-w_{h}\right), \quad \forall v_{h} \in K_{h}, \quad w_{h} \in K_{h} .
$$

Since bilinear form $a(\cdot, \cdot)$ is symmetric, (4.1) is actually equivalent to the quadratic programming problem:

$$
\min _{v \in K_{h}}\left[\frac{1}{2} a(v, v)-(g, v)\right]
$$

Let $\left\{\phi_{j}\right\}_{j=1 \cdots M}$ be a basis of $V_{h}$ with linear functions such that $\phi_{j}(x) \geq 0, \forall x \in \Omega$ and satisfying

$$
\phi_{j}\left(x_{i}\right)= \begin{cases}1, & i=j \\ 0, & i \neq j\end{cases}
$$

where $x_{i}$ is a node of the finite element mesh. 
Then (4.2) reduces to the following vector form:

$$
\min _{z \geq 0}\left[\frac{1}{2} z^{\prime} D z-P^{\prime} z\right]
$$

where $z \geq 0$ means componentwise inequality. Here, $D=\left(d_{i j}\right)$ with $d_{i j}=\left(\nabla \phi_{i}, \nabla \phi_{j}\right)$, $1 \leq i, j \leq M$, and $z$ is the coefficient vector with $\left\{\phi_{j}\right\}$ corresponding to the function $v$ in (4.2). Also, $P \equiv\left(\left(g, \phi_{j}\right)\right)_{1 \leq j \leq M}$.

By the well-known result (see, for example, Bazaraa and Shetty 1979), a vector $z=\left(z_{j}\right) \in R^{M}$ with $z \geq 0$ is an optimal solution to (4.2) if and only if there exists $u=\left(u_{j}\right) \in R^{M}$ such that

$$
\left\{\begin{array}{l}
u-D z=-P \\
u z=0, \quad u \geq 0, \quad z \geq 0
\end{array}\right.
$$

Here, $u z$ means inner product on $R^{M}$.

By the fact that $u, z \geq 0$, we have $z_{j}=0$ or $u_{j}=0$ for $1 \leq j \leq M$. Thus we have the following system of nonlinear equations:

$$
\left\{\begin{array}{l}
u-D z=-P \\
u_{j} z_{j}=0, \quad 1 \leq j \leq M
\end{array}\right.
$$

in $2 M$ unknowns $u, z$.

Now let $A_{j}$ be intervals on $R^{1}, 1 \leq j \leq M$, and let $\sum_{j=1}^{M} A_{j} \phi_{j}$ be a linear combination of $\left\{\phi_{j}\right\}$, i.e., an element of the power set $2^{V_{h}}$ in the following sense:

$$
\sum_{j=1}^{M} A_{j} \phi_{j}=\left\{\sum_{j=1}^{M} a_{j} \phi_{j} ; a_{j} \in A_{j}, \quad 1 \leq j \leq M\right\} .
$$

Now, we denote the set of all nonnegative real numbers by $R^{+}$and for any $\alpha \in R^{+}$, we set

$$
[\alpha] \equiv\left\{\phi \in K_{h}^{*} ; \quad\|\phi\|_{V} \leq \alpha\right\} .
$$

Let denote all the set of linear combinations of $\left\{\phi_{j}\right\}$ with interval coefficients by $\mathcal{D}$, i.e.,

$$
\mathcal{D} \equiv\left\{\sum_{j=1}^{M} A_{j} \phi_{j} \mid A_{j} ; \text { interval in } R^{1}, \quad 1 \leq j \leq M\right\} .
$$

For $(w, \alpha) \in \mathcal{D} \times R^{+}$, setting $W:=w \bigoplus[\alpha]$ and $g=f(W)$ in (2.1), we consider the following nonlinear system:

$$
\left\{\begin{array}{l}
u-D z=-\left(f(W), \phi_{j}\right), \quad 1 \leq j \leq M \\
u_{j} z_{j}=0, \quad 1 \leq j \leq M
\end{array}\right.
$$

Here, $\left(f(W), \phi_{j}\right)$ is evaluated as interval $Y_{i}$ such that $\left\{\left(f(w), \phi_{j}\right) \mid w \in W\right\} \subset Y_{i}$.

In order to solve (4.7) with guaranteed accuracy, we use the following theorem which is given in Rump (1983). 
THEOREM 4.1. Let $\Phi: R^{n} \rightarrow R^{n}$ be a function with continuous first derivative and let $R \in R^{n \times n}$ (real $n \times n$ matrix), $\quad \tilde{x} \in R^{n}$. Denote the Jacobian matrix of $\Phi$ by $\Phi^{\prime} \in R^{n \times n}$ and for $X \in I R^{n}$ (real interval vectors with $n$ components) define $\Phi^{\prime}(X):=\cap\left\{Y \in I R^{n}: \Phi^{\prime}(x) \in Y\right.$ for all $\left.x \in X\right\}$. If for some $X \in I R^{n}$ with $0 \in X$

$$
-R \cdot \Phi(\tilde{x})+\left\{I-R \cdot \Phi^{\prime}(\tilde{x}+X)\right\} \cdot X \subseteq \stackrel{0}{X}
$$

then there exists an $\hat{x} \in \tilde{x}+\stackrel{\circ}{X}$ with $\Phi(\hat{x})=0$.

Using the solution of (4.7), we define the map $T: V \rightarrow \mathcal{D}$ by

$$
T(W)=\widetilde{W}
$$

where $\widetilde{W}$ stands for the set of verified solutions obtained by Theorem 4.1 ; that is, an interval vector, of the nonlinear system (4.7) with interval coefficients in the right hands sides.

We now consider the fully automatic computer generation of the set $W$ satisfying Lemma 3.2. First, we generate the following iteration sequence $\left\{\left(w_{h}^{(n)}, \alpha_{n}\right)\right\}$ for $n=$ $0,1,2, \cdots$, where $\left(w_{h}^{(n)}, \alpha_{n}\right) \in \mathcal{D} \times R^{+}$with $w_{h}^{(n)} \subset K_{h}$ and set $W^{(n)}:=w_{h}^{(n)} \bigoplus\left[\alpha_{n}\right]$.

For $i=0$, we choose an appropriate initial value $w_{h}^{(0)} \in K_{h}$ and $\alpha_{0} \in R^{+}$. Usually, $w_{h}^{(0)}$ will be determined as

$$
a\left(w_{h}^{(0)}, v_{h}-w_{h}^{(0)}\right) \geq\left(f\left(w_{h}^{(0)}\right), v_{h}-w_{h}^{(0)}\right), \forall v_{h} \in K_{h}, w_{h}^{(0)} \in K_{h}
$$

which corresponds to the Galerkin approximation for (2.3). And the standard selection for $\alpha_{0}$ will be $\alpha_{0}=0$. For $n \geq 1$, first for a given $0<\delta \ll 1$, we define the $\delta$-inflation of $\left(w_{h}^{(n-1)}, \alpha_{n-1}\right)$ by

$$
\left\{\begin{array}{l}
\widetilde{w}_{h}^{(n-1)}=w_{h}^{(n-1)}+\sum_{j=1}^{M}[-1,1] \delta \phi_{j} \\
\widetilde{\alpha}_{n-1}=\alpha_{n-1}+\delta
\end{array}\right.
$$

Next, for the set $\widetilde{W}^{(n-1)}=\widetilde{w}_{h}^{(n-1)} \bigoplus\left[\widetilde{\alpha}_{n-1}\right]$, we define $w_{h}^{(n)} \in \mathcal{D}$ and $\alpha_{n} \in R^{+}$by

$$
\left\{\begin{array}{l}
w_{h}^{(n)}=T\left(\widetilde{W}^{(n-1)}\right), \\
\alpha_{n}=C h \sup _{w \in \widetilde{W}(n-1)}\|f(w)\|_{L^{2}(\Omega)} .
\end{array}\right.
$$

Here, $C$ is the constant defined in (2.6).

Now we have the following verification condition on a computer.

THEOREM 4.2. If for an integer $N$, two relationships

$$
w_{h}^{(N)} \subset \widetilde{w}_{h}^{(N-1)} \text { and } \alpha_{N}<\widetilde{\alpha}_{N-1}
$$

hold, then there exists a solution $w$ of $(2.3)$ in $w_{h}^{(N)} \bigoplus\left[\alpha_{N}\right]$. Here, the first term of (4.11) means the inclusion in the sense of each coefficient interval of $w_{h}^{(N)}$ and $\widetilde{w}_{h}^{(N-1)}$

We omit the proof of this theorem, for it is quite similar to that of the corresponding theorem in Nakao (1992). 
Although the verification method in the above enables us to find a solution in the set $w_{h}^{(N)} \bigoplus\left[\alpha_{N}\right]$, it is impossible to assure uniqueness of the solution in the same set. We now present a technique including the verification of uniqueness under the following additional assumption.

A4. Suppose that there exists a $\lambda<1$ such that

$$
\left\|P_{K} F\left(w_{1}\right)-P_{K} F\left(w_{2}\right)\right\|_{V} \leq \lambda\left\|w_{1}-w_{2}\right\|_{V}, \forall u_{1}, w_{2} \in W
$$

By using above assumption and Banach fixed point theorem, we have the following direct consequence which sharpen the Theorem 4.2.

Theorem 4.3. Assume that $P_{K} F$ satisfies $\mathrm{A} 4$ on a set $W$. If for an integer $N$, two relationships

$$
w_{h}^{(N)} \subset \widetilde{w}_{h}^{(N-1)} \text { and } \alpha_{N}<\widetilde{\alpha}_{N-1}
$$

hold, then there is one and only one solution $w$ of (2.3) in $w_{h}^{(N)} \bigoplus\left[\alpha_{N}\right]$.

\section{Numerical examples}

In this section, we present some numerical examples for verification according to the procedures described in the previous section. We consider the case

$$
f(w)=a w+b .
$$

Here, we assume that $a, b \in L^{\infty}(\Omega)$. Frist, in order to validate A4, we need some properties for $P_{K} F$. Let $\mathcal{L}(V)$ be the set of bounded linear operators from $V$ to $V$.

We consider the following eigenvalue problem:

$$
\left\{\begin{array}{l}
-\Delta u=\lambda u \text { in } \Omega \\
u=0, \text { on } \partial \Omega
\end{array}\right.
$$

As well-known, the first eigenvalue $\lambda_{1}$ of $(5.2)$ is equivalent to the the following problem

$$
\min _{w \in V} \frac{\|w\|_{V}^{2}}{\|w\|_{L^{2}}^{2}}=\lambda_{1}
$$

Hence, we obtain

$$
\forall w \in V, \quad \frac{\|w\|_{V}}{\|w\|_{L^{2}}} \geq \sqrt{\lambda_{1}} .
$$

Furthermore, by well-known results, it follows that

$$
\lambda_{1}=\frac{1}{2 \pi^{2}}
$$

for the unit square in two dimensional case. In one dimensional case, we can take $\lambda_{1}$ as

$$
\lambda_{1}=\frac{1}{\pi^{2}} \text {. }
$$

And, by (2.7), we have $F\left(w_{1}\right)-F\left(w_{2}\right)=(-\Delta)^{-1}\left(a\left(w_{1}-w_{2}\right)\right)$. 
Now, setting $A w:=(-\Delta)^{-1} a w$, consider the following inequality:

$$
\begin{aligned}
& \left\|P_{K} F\left(w_{1}\right)-P_{K} F\left(w_{2}\right)\right\|_{V} \\
& \leq\left\|F\left(w_{1}\right)-F\left(w_{2}\right)\right\|_{V} \\
& \leq\left\|A\left(w_{1}-w_{2}\right)\right\|_{V} \\
& \leq\|A\|_{\mathcal{L}(V)}\left\|w_{1}-w_{2}\right\|_{V} .
\end{aligned}
$$

Here, we used the fact that $\left\|P_{K}\right\|_{\mathcal{L}(V)} \leq 1$. Further, we obtain

$$
\begin{aligned}
\|A\|_{\mathcal{L}(V)} & =\sup _{w \neq 0 \in V} \frac{\|A w\|_{V}}{\|w\|_{V}}=\sup _{w \in V} \frac{(\nabla A w, \nabla A w)}{\|w\|_{V}\|A w\|_{V}} \\
& =\sup _{w \in V} \frac{(a w, A w)}{\|w\|_{V}\|A w\|_{V}} \leq\|a\|_{L^{\infty}} \sup _{w \in V} \frac{\|w\|_{L^{2}}\|A w\|_{L^{2}}}{\|w\|_{V}\|A w\|_{V}}
\end{aligned}
$$

Hence, by using (5.3) and (5.4), we have

$$
\|A\|_{\mathcal{L}(V)} \leq \frac{\|a\|_{L^{\infty}}}{2 \pi^{2}}
$$

for the unit square in two dimensional case. Similarly, for one dimensional case, we obtain

$$
\|A\|_{\mathcal{L}(V)} \leq \frac{\|a\|_{L^{\infty}}}{\pi^{2}}
$$

Therefore, we have the following results.

THEOREM 5.1. If the function a in (5.1)satisfies

$$
\frac{\|a\|_{L^{\infty}}}{2 \pi^{2}}<1(n=2) \quad \text { or } \quad \frac{\|a\|_{L^{\infty}}}{\pi^{2}}<1(n=1)
$$

then the assumption $\mathrm{A} 4$ holds.

In what follows we consider the one dimensional case. Then, we can estimate constant $C$ in Section 2 as below.

Let $\Omega=(0,1)$ and let $g \in L^{2}(\Omega)$. For a positive integer $M$, set $h=\frac{1}{M}$; we consider $x_{i}=i \cdot h$ for $i=0,1,2, \cdots M$, that is, a uniform partition of $\Omega$ and set $e_{i}=\left(x_{i-1}, x_{i}\right), \quad i=1,2, \cdots M$. We then approximate $V$ and $K$ by

$$
V_{h}=\left\{v_{h} \in C^{0}(\Omega) ; \quad v_{h}(0)=v_{h}(1)=0,\left.\quad v_{h}\right|_{e_{i}} \in P_{1}, i=1,2,3 \cdots M\right\},
$$

where $P_{1}$ is the space of polynomials of degree $\leq 1$, and

$$
K_{h}=\left\{v_{h} \in V_{h} ; \quad v_{h}(x) \geq 0, \quad \forall x \in \Omega\right\},
$$

respectively.

Regarding the approximation error $\left\|w_{h}-w\right\|_{V}$ and $\left\|w_{h}-w\right\|_{L^{2}(\Omega)}$, we have the following lemma (see Ryoo and Nakao (1998)).

LEMMA 5.2. Let $w$ and $w_{h}$ be solutions of (2.1) and (4.1), respectively. If $g \in L^{2}(\Omega)$, then we have

$$
\left\|w_{h}-w\right\|_{V} \leq \frac{\sqrt{5}}{\pi} h\|g\|_{L^{2}(\Omega)}
$$




$$
\left\|w_{h}-w\right\|_{L^{2}(\Omega)} \leq \frac{\sqrt{5}}{\pi}\left(\frac{1}{\pi}+\frac{4 \sqrt{2}}{3}\right) h^{2}\|g\|_{L^{2}(\Omega)}
$$

Hence, we may take $C=\frac{\sqrt{5}}{\pi}$ in (2.6). We now present some computed results of verification with uniqueness.

Example 1. We consider

$$
f(w)=w \sin x-\sin x \sin 2 \pi x+4 \pi^{2} \sin 2 \pi x .
$$

We choose the basis $\left\{\phi_{i}\right\}_{i=1}^{M}$ of $V_{h}$ as usual hat functions.

The execution conditions are as follows.

$$
\begin{aligned}
& \text { Numbers of elements }=100 \\
& \operatorname{dim} V_{h}=99 \\
& \text { Extension parameters : } \delta=10^{-3} \\
& \text { Initial values : } w_{h}^{(0)}=\text { Galerkin approximation }(4.9), \alpha_{0}=0, \\
& \text { the outline of } w_{h}^{(0)} \text { is displayed in Figure } 1 .
\end{aligned}
$$

In this case, it would be deduced that there exists a free boundary around $x=0.717172$.

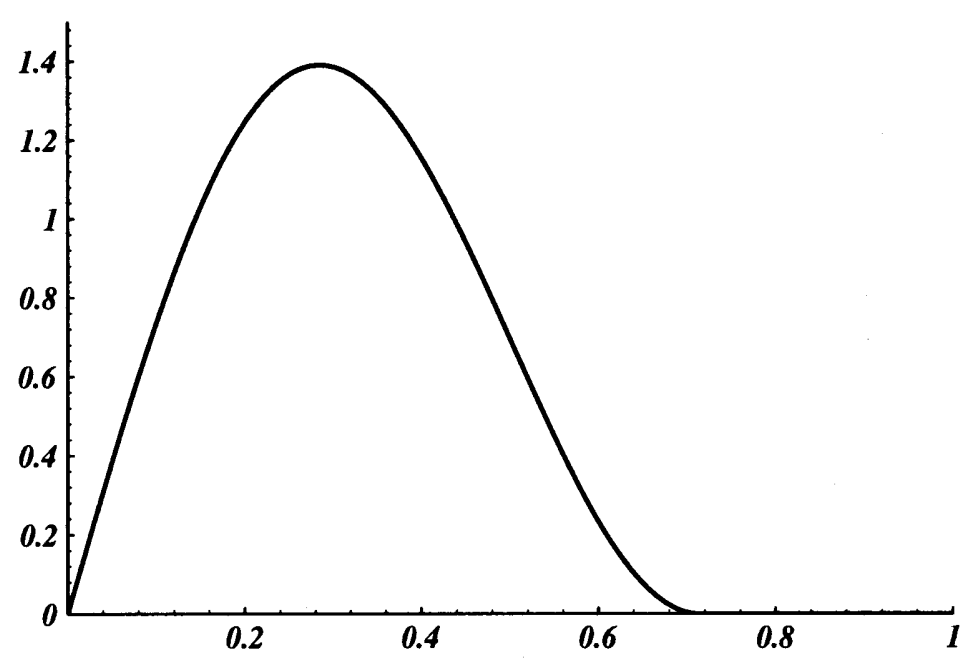

Figure 1: Approximate solution $w_{h}^{(0)}$ 
The results are as follows:

Iteration numbers for verification : $N=3$

$L^{2}$ - error bound : 0.001952

Maximum width of coefficient intervals in $\left\{w_{h}^{(N)}\right\}=0.001707$.

Example 2. Next we consider

$$
f(w)=K w+8 \cos 2 \pi x
$$

where $K$ is a constant. The basis $\left\{\phi_{i}\right\}_{i=1}^{M}$ of $V_{h}$ is the same as in Example 1 .

The execution conditions are as follows.

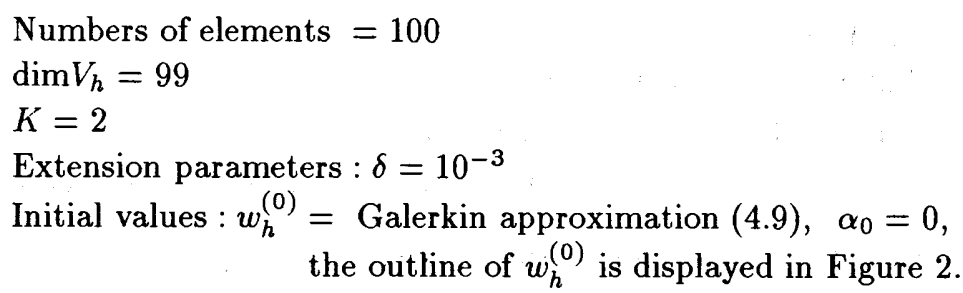

From the Figure 2, two free boundary points can be located around $x=0.367347$ and $x=0.632653$.

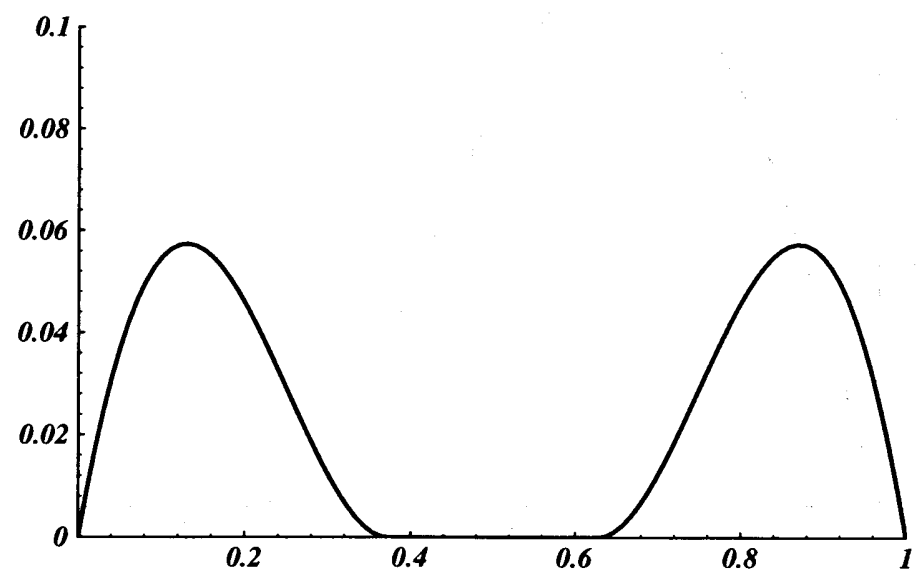

Figure 2: Approximate solution $w_{h}^{(0)}$ 
The results are as follows:

Iteration numbers for verification : $N=3$

$L^{2}$ - error bound : 0.000052

Maximum width of coefficient intervals in $\left\{w_{h}^{(N)}\right\}=0.000127$.

REMARK. In the above calculations, we used usual computer arithmetic with double precision instead of strict interval computions (e.q., ACRITH-XSC, PASCALXSC,C-XSC etc.). But, from our experiences, the order of magnitude of roundoff error is , in general, under $10^{-10}$. Therefore, it is almost negligible compared with the truncation error which amounts to $10^{-3} \sim 10^{-2}$.

\section{Acknowledgement}

The author is deeply grateful to Professor M.T.Nakao for his valuable advices and discussions. Also, the author would like to appreciate very much for the referee's useful comments.

\section{References}

Bazaraa, M.S.and Shetty, C.M, (1979). Nonlinear Programming, John Wiley, New York.

Glowinski, R, (1984). Numerical Methods for Nonlinear Variational Problems, Springer, New York.

Nakao, M.T, (1988). A numerical approach to the proof of existence of solutions for elliptic problems, Japan Journal of Applied Math. 5, 313-332.

Nakao, M.T, (1992). A numerical verification method for the existence of weak solutions for nonlinear boundary value problems, Journal of Math. Analysis and Appl. 164, 489-507.

Nakao, M.T and Ryoo, C.S, (1998). Numerical verifications of solutions for variational inequalities using Newton-like method, (to appear in Proceedings of the 1st Setouchi Symposium on Applied Mathematics, on June 28, 1997, 10 pages.)

Nakao, M.T. and Yamamoto, N, (1998). Numerical verification of solutions for nonlinear elliptic problems using an $L^{\infty}$ residual method, J. Math.Anal. Appl. 217, 246-262.

Rodrigues, J.F, (1987). Obstacle problems in Mathematical Physics, Math.Stud. 134, North-Holland, Amsterdam.

Rump, S.M, (1983). Solving algebraic problems with high accuracy, A new approach to scientific computation, Academic Press, New York.

Ryoo, C.S. and Nakao, M.T, (1998). Numerical verification of solutions for variational inequalities, (to appear in Numerische Mathematik)

Yamamoto, N. and Nakao, M.T, (1993). Numerical verifications of solutions for elliptic equations in nonconvex polygonal domains, Numerische Mathematik, 65, 503-521. 
Yamamoto, N. and Nakao, M.T, (1995). Numerical verifications of solutions to elliptic equations using residual iterations with a higher order finite element, Journal of Comp. and Applied Math. 60, 271-279.

Received September 25, 1997 